\title{
Estimulação cardíaca com variação de freqüência em sistema de alça fechada e sensor de contractilidade miocárdica: avaliação tardia em estudo multicêntrico
}

\author{
José Carlos S. de ANDRADE*; Veridiana Silva de ANDRADE*; Paulo Ceratti AZAMBUJA*; \\ Gilberto Venossi BARBOSA ${ }^{* * *}$; Humberto BENEDETTI*; Roque Paulo Torres FALLEIRO ${ }^{* *}$; \\ Luis Sérgio FRAGOMENI ${ }^{* *}$; José JAZBIKo; Newton José Martins MOTTA ${ }^{\circ 0}$; Aldo Aller TOMAS ${ }^{000}$
}

RBCCV 44205-574

Andrade J C S, Andrade V S, Azambuja P C, Barbosa G V, Benedetti H, Falleiro R P T, Fragomeni L S, Jazbik J, Motta N J M, Tomas A A - Estimulação cardíaca com variação de freqüência em sistema de alça fechada e sensor de contractilidade miocárdica: avaliação tardia em estudo multicêntrico. Rev Bras Cir Cardiovasc 2002; 17(1): 73-8.

RESUMO: Introdução:O tratamento das bradicardias com o emprego de marcapassos (MP) de dupla câmara com resposta de freqüência (DDD,R) tem incentivado a procura de um sensor ideal. Reavaliamos tardiamente (3 anos) a resposta de freqüência desses MP com sensor de contractilidade miocárdica em sistema de alça fechada.

Casuística e Métodos: Do estudo inicial feito em 1997 foram reavaliados 30 pacientes que possuíam doença binodal e 3 anos de implante, sendo $60 \%$ do sexo masculino, com idades de 17-87 anos (média =61). A freqüência cardíaca $(F C)$ foi observada através do Holter de $24 \mathrm{~h}$ e do histograma de freqüência do MP nas atividades diárias e testes de caminhada de 6 minutos e de subir/descer escadas. Determinaramse os limiares crônicos de estimulação e sensibilidade atriais (A) e ventriculares (V), cotejando-os com os obtidos no implante e no pós-operatório imediato.

Resultados: As médias dos limiares no intra-operatório, 30 dias e 3 anos foram as seguintes: estimulação A $(0,8,1,4$ e $1,1 \mathrm{~V})$ e $V(0,5,1,1$ e 1,0V), sensibilidade $A(2,3,2,8$ e $2,6 \mathrm{mV})$ e $V(10,6,6,3$ e $6,3 \mathrm{mV})$ A FC aos 3 anos aumentou nas atividades diárias (físicas e mentais) de 33 a $91 \%$ e nos testes de esforço físico de 21 a $130 \%$, à semelhança dos resultados em 30 dias (teste t de Student e análise de variância). Esse sensor de contractilidade manteve aos 3 anos os bons resultados obtidos aos 30 dias, observando-se, como efeitos adversos: o alto consumo energético em 2 pacientes e a dificuldade de ajuste do sensor em outros dois.

DESCRITORES: Estimulação cardíaca artificial. Marcapasso artificial. Contração miocárdica.

Estudo multicêntrico realizado em:

*Escola Paulista de Medicina. Universidade Federal de São Paulo. São Paulo, SP, Brasil.

${ }^{*}$ Faculdade de Medicina da Universidade de Passo Fundo. Passo Fundo, RS, Brasil.

${ }^{* \star *}$ Faculdade de Medicina da Universidade Federal do Rio Grande do Sul. Porto Alegre, RS, Brasil.

${ }^{\circ}$ Hospital Universitário Pedro Ernesto. Rio de Janeiro, RJ, Brasil.

oo Hospital Santa Isabel. Blumenau, SC, Brasil.

${ }^{000}$ Casa de Saúde São José. Uberaba, MG, Brasil.

Apresentado no 28을 Congresso Nacional de Cirurgia Cardíaca. Ouro Preto, MG, 5 a 7 abril de 2001.

Endereço para correspondência: José Carlos S. Andrade. Rua Borges Lagoa, 783-5ํo andar. São Paulo, SP, Brasil. CEP: 04038-031. Tel: (11) 557390.

Fax: (11) 55730303.e-mail: cardiovascular.dcir@epm.br 
Andrade J C S, Andrade V S, Azambuja P C, Barbosa G V, Benedetti H, Falleiro R P T, Fragomeni L S, Jazbik J, Motta N J M, Tomas A A - Estimulação cardíaca com variação de freqüência em sistema de alça fechada e sensor de contractilidade miocárdica: avaliação tardia em estudo multicêntrico. Rev Bras Cir Cardiovasc 2002; 17(1): 73-8.

\section{INTRODUÇÃO}

A eliminação dos sintomas dos pacientes portadores de bloqueio atrioventricular avançado e a redução de sua mortalidade foram objetivos alcançados pelas primeiras gerações de marcapassos. A observação, entretanto, de que esses pacientes mantinham-se diuturnamente com a mesma FC, sem adaptação às demandas metabólicas dos esforços e, por vezes, com baixa capacidade física, fez com que a estimulação cardíaca artificial corresse atrás da resposta de freqüência e da sincronização atrioventricular.

A preocupação com o desempenho hemodinâmico oferecido pela variação da freqüência cardíaca e pelo sincronismo da contração atrial e ventricular surgiu já no advento dos marcapassos implantáveis. NATHAN et al. (1), em 1963, relatavam a utilização de marcapassos que estimulavam o ventrículo após captarem a onda de ativação elétrica atrial. Entretanto, a necessidade do acesso epicárdico atrial e ventricular, acrescidos do grande consumo de energia de tais geradores, manteve esse tipo de estimulação praticamente na experimentação clínica. Seu retorno triunfal ocorreu na década de 80, após os enormes avanços ocorridos na tecnologia tanto de fabricação dos marcapassos, com a utilização de circuitos integrados, baterias de lítio, fechamento hermético dos geradores, multiprogramabilidade, telemetria bidirecional, como na dos cabos-eletrodos, desenvolvidos com menor calibre, maior flexibilidade, maior resistência, histocompatibilidade e de fácil implantação em átrio e ventrículo direitos através da via venosa (2-4).

A utilização clínica maciça da estimulação cardíaca bicameral (DDD), dita "fisiológica", coincidiu com a ampliação das indicações de implante, dado o estágio avançado dos conhecimentos eletrofisiológicos e da fisiopatologia dos distúrbios da condução cardíaca. Surgiu, então, a necessidade de se solucionar o problema dos pacientes que apresentavam bloqueio atrioventricular total (BAVT) com fibrilação atrial (FA) de baixa freqüência ou com disfunção sinusal: parada sinusal (PS), bloqueio sinoatrial (BSA), bradicardia sinusal ou mesmo incompetência cronotrópica do nó sinusal, nos quais ficava impraticável a utilização do guia de freqüência do próprio nó sinusal.

Foi a fase em que os entusiastas convencidos da importância da variabilidade da freqüência cardíaca no ajuste do débito cardíaco (DC) diante da demanda metabólica física ou mental, aplicaram seus esforços no desenvolvimento dos marcapassos com biossensores ou marcapassos com resposta de freqüência ou, ainda, marcapassos responsivos ${ }^{(5)}$.

O organismo humano, em situação de esforço, de aumento metabólico, modifica, além da freqüência atrial, vários outros parâmetros, tais como: freqüência respiratória, $\mathrm{pH}$ sangüíneo, temperatura, volume sistólico, pressão ventricular, intervalo QT, saturação sangüínea de oxigênio, movimentação corporal, contractilidade cardíaca etc.

A monitorização de um desses parâmetros, ditos indicadores de demanda metabólica, permite a construção de um MP, que, diante de um esforço, realize a necessária adaptação na freqüência de estimulação.

O esquema de funcionamento é observado na Figura 1, ou seja, com o exercício são alterados os valores do indicador; essas alterações são captadas e quantificadas por um sensor, o

\section{CONCEITO DE MP RESPONSIVO}

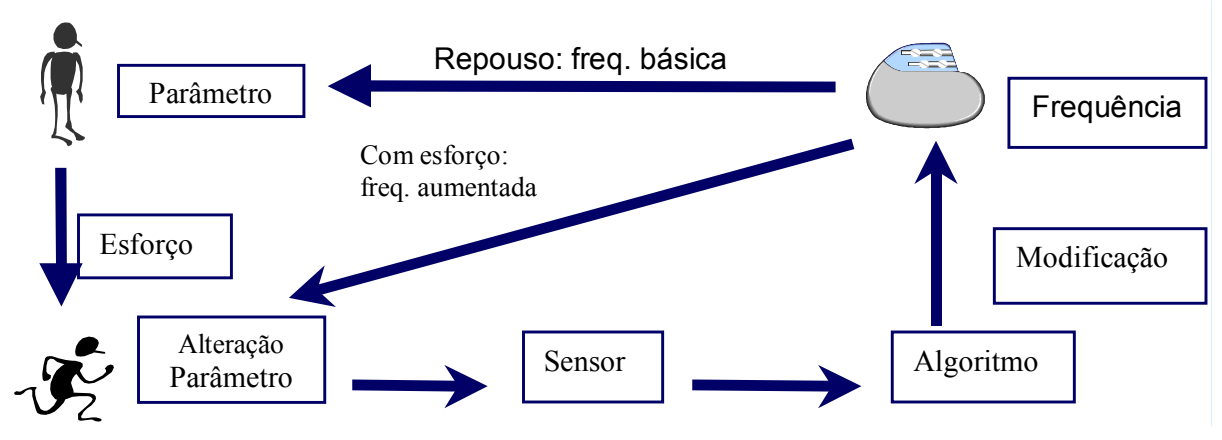

Fig. 1 - Esquema básico do funcionamento do MP responsivo, onde as alterações geradas pelo esforço são registradas pelo sensor que modifica a freqüência de estimulação do MP, adaptando-a ao esforço produzido. 
Andrade J C S, Andrade V S, Azambuja P C, Barbosa G V, Benedetti H, Falleiro R P T, Fragomeni L S, Jazbik J, Motta N J M, Tomas A A - Estimulação cardíaca com variação de freqüência em sistema de alça fechada e sensor de contractilidade miocárdica: avaliação tardia em estudo multicêntrico. Rev Bras Cir Cardiovasc 2002; 17(1): 73-8.

resultado é trabalhado com um algoritmo e promove alteração na freqüência de estimulação do MP, procurando adaptá-lo à demanda metabólica requerida. É fundamental que o conjunto indicador de demanda metabólica, sensor, algoritmo e variador de freqüência possua algumas características: especificidade, proporcionalidade, boa velocidade de resposta, durabilidade, confiabilidade, programabilidade e segurança. Outra característica pretendida na estimulação cardíaca atual é que o sistema ofereça uma variação circadiana da freqüência de estimulação, ou seja, uma variação estreitamente relacionada com a atividade, física ou mental, diurna ou noturna, do paciente. Para isso, o indicador escolhido deve estar sob influência do sistema nervoso autônomo (SNA).

No organismo humano, o DC é continuamente regulado pelo SNA, a fim de que as variações da demanda hemodinâmica sejam prontamente atendidas. Como a atividade cardíaca está intimamente relacionada ao eixo neuro-humoral, a liberação e o efeito de substâncias como as catecolaminas são de fundamental importância.

A observação da Figura 2 mostra que o DC é dependente de duas variáveis; a FC e o volume

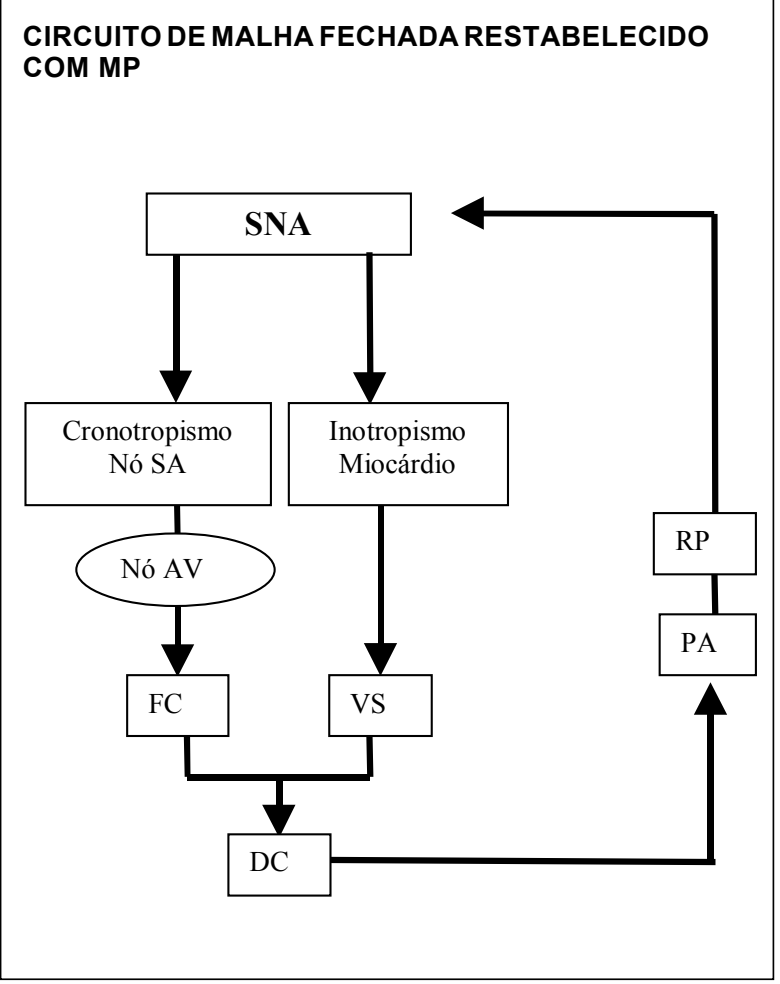

Fig. 2 - Esquema do controle autônomo do DC através da FC (via cronotrópica) e VS (via inotrópica). sistólico (VS). Ambos são regulados pelo SNA através de duas vias: a cronotrópica e a inotrópica, realimentadas constantemente pelas informações dadas pelas variações da pressão sangüínea arterial média ( $P A)$ e da resistência periférica total (RP), frutos da própria variação do DC, constituindo um circuito de malha fechada.

Nas doenças do sistema de condução cardíaca, em que haja perda ou limitação na geração de impulsos pelo nó sinusal ou na condução atrioventricular, a via cronotrópica fica interrompida e a regulação do DC restrita às alterações do retorno venoso e da contractilidade miocárdica, insuficientes para a manutenção de uma fisiologia normal. Entretanto, nessa situação patológica, o estado de contractilidade cardíaca continua refletindo diretamente a influência autonômica. Assim sendo, a sua monitoração permite obter um ótimo parâmetro para o restabelecimento artificial do controle, em maIha fechada, da FC $(6,7)$.

O implante de um marcapasso com sensor de contractilidade cardíaca possibilita, então, o restabelecimento da influência autonômica na regulação da FC (Figura 3) e, portanto, da variabilidade da FC frente às demandas metabólicas.

\section{CIRCUITO FISIOLÓGICO DE MALHA FECHADA}

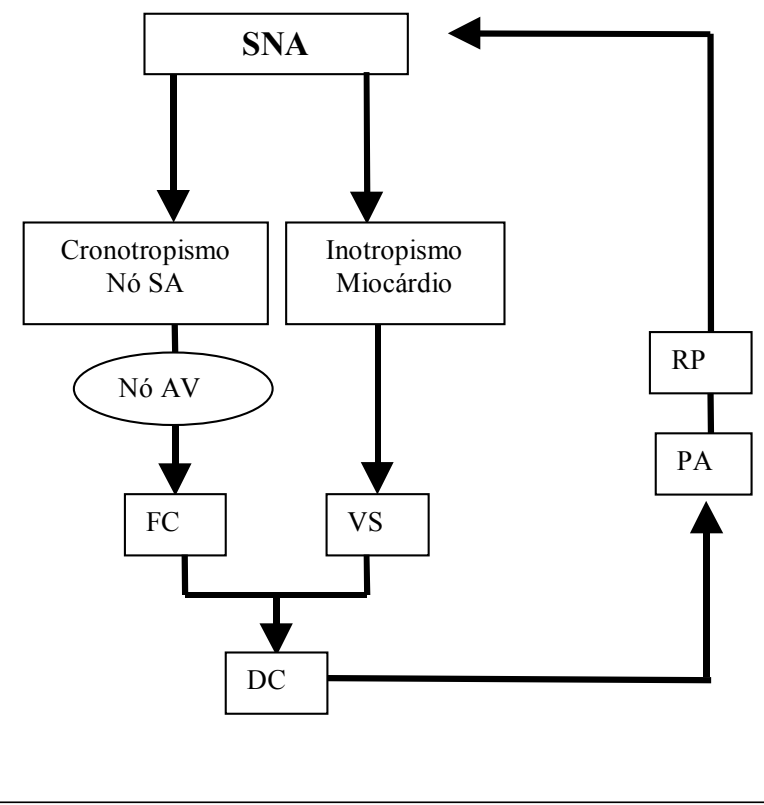

Fig. 3 - Interrompida a via cronotrópica por doença de nó sinusal ou por Bloqueio AV, o restabelecimento da variação da FC é feito artificialmente com o MP, via inotropismo cardíaco. 
Andrade J C S, Andrade V S, Azambuja P C, Barbosa G V, Benedetti H, Falleiro R P T, Fragomeni L S, Jazbik J, Motta N J M, Tomas A A - Estimulação cardíaca com variação de freqüência em sistema de alça fechada e sensor de contractilidade miocárdica: avaliação tardia em estudo multicêntrico. Rev Bras Cir Cardiovasc 2002; 17(1): 73-8.

Há 3 anos, num estudo multicêntrico(8), foi feita uma avaliação desse sistema de estimulação em pacientes com doença binodal, aos 30 dias do implante. Os resultados mostraram que o MP possibilitava a variação da FC, durante esforços físicos de 5 a $128 \%$ e durante esforços mentais de 5 a $80 \%$.

Este trabalho reavalia esses pacientes com 0 objetivo de analisar a manutenção ou não desses resultados em longo prazo: 3 anos de evolução.

\section{CASUÍSTICA E MÉTODOS}

Do estudo multicêntrico brasileiro inicial (8) feito com o emprego de um sistema de estimulação cardíaca tipo DDD, $R$ em pacientes com insuficiência cronotrópica bicameral, foram reestudados aqueles com 3 anos de evolução. Em número de 30 , sendo 18 do sexo masculino e 12 do feminino, com idades variando de 17 a 87 anos (média de 61), todos apresentavam doença do nó sinusal (DNS) e BAVT.

A freqüência do MP foi observada nas atividades diárias habituais de cada paciente, no teste de caminhada de 6 minutos e no teste de subir e descer escadas. As mensurações dessas freqüências foram feitas com o registro da eletrocardiografia dinâmica (Holter) por 24 horas e com o histograma de freqüência obtido telemetricamente do próprio MP.

Por ocasião desses registros, foram também determinados os limiares crônicos de estimulação e de sensibilidade tanto na câmara atrial, como na ventricular, cotejando-se esses valores com os obtidos tanto durante o implante, como no pósoperatório de 30 dias.

\section{Análise Estatística}

Para comparação das médias, foi utilizado o teste t de Student e, para comparações nos grupos de variáveis, a análise de variância de dados repetidos, sendo definida significância estatística para os valores de $p$ menor ou igual a 0,05.

\section{RESULTADOS E COMENTÁRIOS}

\section{Limiares de Estimulação e Sensibilidade}

Em trabalho anterior, a média de limiares agudos de estimulação na configuração unipolar foi de $0,82 \pm 0,45$ e $0,55 \pm 0,43 \mathrm{~V}$, respectivamente, para átrios e ventrículos; aos 30 dias, esses valores foram de $1,44 \pm 0,68 \mathrm{~V}$ para átrios e $1,18 \pm 0,71 \mathrm{~V}$ para ventrículos. Repetidas essas determinações aos 3 anos de evolução, constataram-se os valores de $1,10 \pm 0,58 \vee$ para átrios e $1,00 \pm 0,40 \mathrm{~V}$ para ventrículos. Observa-se, portanto, que os limiares de estimulação, elevados em cerca de $100 \%$ aos 30 dias (ocasião em que a maturidade da interface eletrodo-coração não era total), ficaram estabilizados, aos 3 anos, em valores inferiores (melhores) aos de 30 dias, mas cerca de 37 a 100\% mais elevados do que os obtidos intra-operatoriamente.

Resultados semelhantes foram observados nos limiares de sensibilidade obtidos no intra-operatório, 30 dias e 3 anos tanto a nível atrial $(2,3 ; 2,8$ e $2,6 \mathrm{mV})$ como a nível ventricular $(10,6 ; 6,3$ e $6,3 \mathrm{mV})$. Estes valores, muito semelhantes com 30 dias e 3 anos, mostram maior estabilidade da captação da atividade elétrica cardíaca frente à maturação da interface eletrodo-coração.

Esses resultados todos são superponíveis a experiências anteriores, já que os cabos eletrodos utilizados são convencionais.

\section{Freqüência de Estimulação}

A análise da freqüência de estimulação feita tanto nos histogramas de freqüência obtidos da memória do gerador, como nos traçados da eletrocardiografia dinâmica, mostrou uma variação de 33 a $91 \%$ nas atividades diárias e de 21 a $130 \%$ durante a realização dos testes programados.

Esses valores são bem próximos dos obtidos há 3 anos, quando, nos testes de atividade mental, a variação chegou a $80 \%$ e, nos testes de atividade física, atingiu $128 \%{ }^{(8)}$.

Além da nítida correlação da freqüência com o nível de atividade física desenvolvida, observou-se que a variação da FC também foi considerável em situações de relativo repouso físico, mas que envolveram momentos de estresse mental como emoções, sustos e exercícios mentais (Figura 4).

A simples observação gráfica tanto dos traçados de Holter, como dos histogramas do MP, mostra uma nítida variação circadiana da freqüência do MP; por outro lado, as oscilações dessa freqüência, constatadas até durante o sono, documentam o interrelacionamento do sensor a influências do SNA, uma das qualidades deste MP (Gráfico 1).

O coração, como órgão central de resposta do sistema cardiovascular, tem resposta quase imediata às requisições do sistema nervoso central. Este atua diretamente no mesmo através de 2 vias: a cronotrópica e a inotrópica, ou seja, através da freqüência cardíaca e do tônus miocárdico (Figura 2). Embora ambas alterações sejam importantes no ajuste do débito cardíaco, a modificação da freqüência, durante o exercício, tem maior expressão 
Andrade J C S, Andrade V S, Azambuja P C, Barbosa G V, Benedetti H, Falleiro R P T, Fragomeni L S, Jazbik J, Motta N J M, Tomas A A - Estimulação cardíaca com variação de freqüência em sistema de alça fechada e sensor de contractilidade miocárdica: avaliação tardia em estudo multicêntrico. Rev Bras Cir Cardiovasc 2002; 17(1): 73-8.

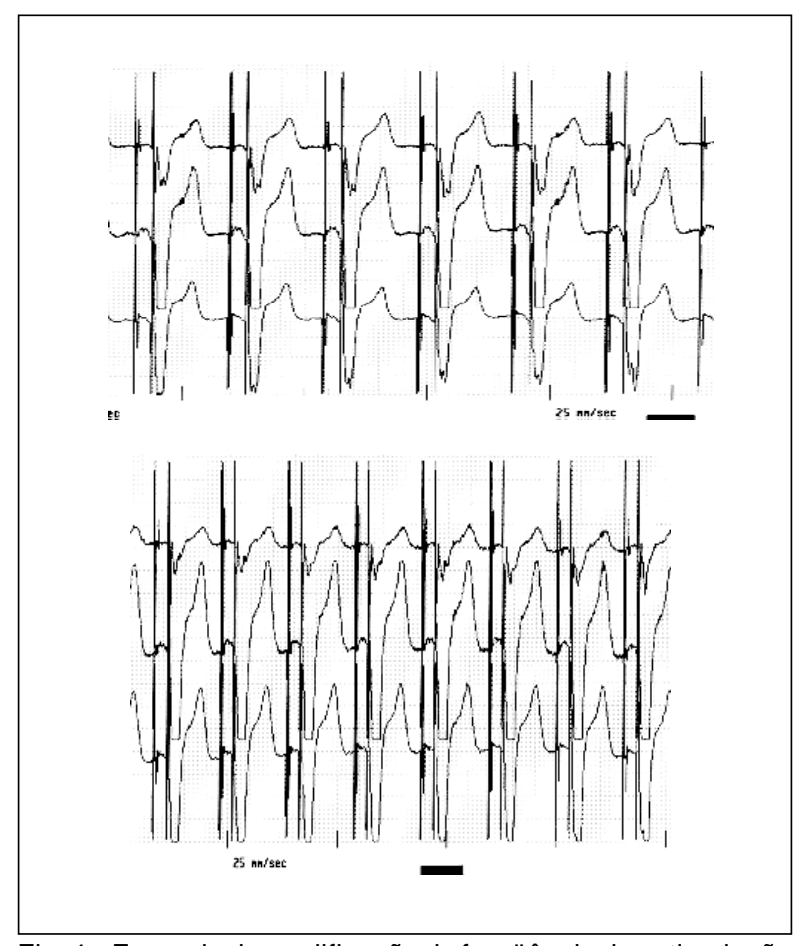

Fig. 4-Exemplo da modificação de freqüência de estimulação ocorrido diante de estresse mental.

\section{GRÁFICO 1 \\ HISTOGRAMA DE FREQÜÊNCIA OBTIDO DO PRÓPRIO MARCAPASSO ATRAVÉS DE TRANS- MISSÃO TELEMÉTRICA}

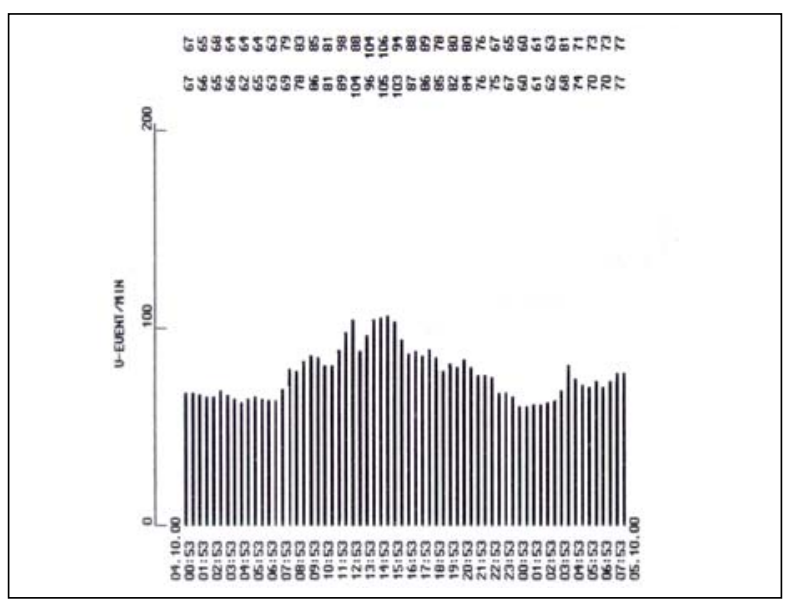

$(9,10)$. Apesar disso, as variações da freqüência e do tônus cardíaco estão intimamente relacionadas.

Disso decorre a grande vantagem do sensor de contractilidade, que, monitorando as alterações do tônus cardíaco, possibilita o ajuste adequado da freqüência de estimulação, o que pode ser observado tanto em solicitações físicas, como mentais.
Já foi demonstrado que essas variações de freqüência desencadeadas pelo MP são semelhantes às de indivíduos normais (11), desde que não se limita o valor máximo ("upper rate") do sensor.

Outra qualidade desse sistema de estimulação é que ele utiliza, como indicador para resposta de freqüência, um parâmetro do próprio controle cardiovascular: a contractilidade cardíaca, medida a partir do registro da impedância cardíaca feito no pólo distal do cabo-eletrodo ventricular, tendo a carcaça do gerador como outro pólo. As variações da impedância obtidas nessa configuração são ocasionadas, principalmente, por mudanças da condutividade em torno do eletrodo distal. Devido às mudanças da contractilidade cardíaca e do volume de sangue e da própria massa miocárdica em torno do eletrodo, ocorridas durante as fases de contração isovolumétrica e ejeção, a condutividade apresenta-se variável, refletindo as mudanças tônicas e geométricas do miocárdio. O registro da impedância constitui, então, um parâmetro que se correlaciona muito bem com a contractilidade cardíaca e faz com que o sensor de contractilidade não tenha o inconveniente de poder, como em outros sensores, realizar uma estimulação com freqüência elevada num coração com baixa fração de ejeção, o que acentuaria a disfunção miocárdica. Este sensor, pelo contrário, à medida em que houver uma diminuição do tônus miocárdico, ele diminuirá também a freqüência de estimulação.

Como comentários adicionais há que se registrar a dificuldade de ajuste do sensor constatada em 2 pacientes, que necessitaram de reprogramações e de limitações no "upper rate" para que não ocorresse elevada freqüência de estimulação. Ambos fatos ocorreram na primeira série de produção desses geradores: os modelos INOS DR.

Também nessa série há que se registrar o alto consumo energético da bateria observado em 2 pacientes, que, por desgaste de bateria, tiveram seus geradores substituídos aos 33 e 38 meses pós implante.

Apesar de serem indesejáveis, esses fatos não depõem nada contra o desempenho do sensor, que confere ao MP uma resposta de freqüência rápida e muito semelhante à do SNA de indivíduos normais, tanto no acompanhamento imediato ao implante, como na observação tardia agora realizada.

\section{CONCLUSÃO}

O sensor de contractilidade cardíaca em sistema de alça fechada manteve, aos 3 anos de utilização, os bons resultados obtidos aos 30 dias. 
Andrade J C S, Andrade V S, Azambuja P C, Barbosa G V, Benedetti H, Falleiro R P T, Fragomeni L S, Jazbik J, Motta N J M, Tomas A A - Estimulação cardíaca com variação de freqüência em sistema de alça fechada e sensor de contractilidade miocárdica: avaliação tardia em estudo multicêntrico. Rev Bras Cir Cardiovasc 2002; 17(1): 73-8.

RBCCV 44205-574

Andrade J C S, Andrade V S, Azambuja P C, Barbosa G V, Benedetti H, Falleiro R P T, Fragomeni L S, Jazbik J, Motta N J M, Tomas A A - Cardiac pacing with frequency variation in closed loop system and myocardial contractility sensor: late evaluation in multicenter study. Rev Bras Cir CardiovasC 2002; 17(1): 73-8.

ABSTRACT: Introduction: The treatment of bradycardias with bicameral pacemakers (PM) with frequency response $(D D D, R)$ has motivated the search of an ideal sensor. We did a late re-evaluation (3 years) the frequency response of those PM with myocardial contractility sensor in closed loop system.

Material and Methods: Thirty patients (pts) of the initial study done in 1997, who presented binodal disease and 3-year implantation were evaluated, being 60\% male, ages between 17-87 (average = 61). Their heart frequency (HF) was observed through a 24 Holter monitoring and MP frequency histogram on the daily activities, plus 6-minute walk and going up and downstairs tests. Chronic pacing thresholds were determined as well as atrial (A) and ventricular (V) sensitivity, checking them against those obtained in the implantation and immediate post-operative periods.

Results: The average threshold in the intra-operative, 30 days and 3 years were as follows: A pacing $(0.8,1.4$ and $1.1 \mathrm{~V})$ and $\mathrm{V}(0.5,1.1 \mathrm{e} 1.0 \mathrm{~V})$, A sensitivity $(2.3,2.8$ e $2.6 \mathrm{mV})$ and $\mathrm{V}(10.6,6.3$ and $6.3 \mathrm{mV})$. The HF at the $3^{\text {rd }}$ year increased for daily activities (physical and mental) from 33 to $91 \%$, and for physical exercises tests from 21 to $130 \%$, similarly to the results obtained in 30 days (test $t$ student and variation analysis). This contractility sensor kept at the 3 rd year the same good results obtained at the 30th day, presenting the following adverse effects: high-energy consumption in 2 pts and difficulty of sensor adjustment in other 2 .

DESCRIPTORS: Cardiac pacing, artificial. Pacemaker, artificial. Myocardial contraction.

\section{REFERÊNCIAS BIBLIOGRÁFICAS}

1 Nathan D A, Center S, Wu C Y, Keller W - An implantable synchronous pacemaker for the longterm correction of complete heart block. Circulation $1963 ; 27: 682-5$

2 Parsonnet V, Furman S, Smyth N P, Bilitch M - Optimal resources for implantable cardiac pacemakers. Circulation 1983; 68:226-44.

3 Bernstein A D, Brownlee R R, Fletcher R, Gold R D, Smith N P D, Spielman S R - Report of the NASPE Mode Committee. Pacing Clin Electrophysiol 1984; 7(3Pt 1): 395-402.

4 Hauser R G, Wimer E A, Timmis G C et al. - Twelve years of clinical experience with lithium pulse generators. Pacing Clin Electrophysiol 1986;9 (6 Pt 2); 1277-81.

5 Andrade J C S - Marcapasso com biosensores. Rebrampa 1988; 1: 32-8.

6 Schaldach $\mathrm{M}$ \& Hutten $\mathrm{H}$ - Intracardiac impedance to determine sympathetic activity in rate responsive pacing. Pacing Clin Electrophysiol 1992;15 (11 Pt 2): 1778-86.
7 Andrade J C S, Greco O T, Tomas A A et al. - Avaliação do sensor de contratilidade cardíaca em sistema de estimulação DDDR: estudo multicêntrico. Reblampa 1997; 10: 220.

8 Andrade J C S, Andrade V S, Buffolo E et al. - Avaliação do sensor de contratilidade cardíaca em sistema DDDR: estudo multicêntrico. Rev Bras Cir Cardiovasc 1998; 13:340-50.

9 Benditt D G, Mianulli M, Fetter J et al. - Single-chamber pacing with activity-initiated chronotropic response: evaluation by cardiopulmonary exercise testing. Circulation 1987; 75: 184-91.

10 Sedney M I, Weijers E, van der Wall E E et al. Short-term and long-term changes of left ventricular volumes during rate-adaptive and single-rate pacing. Pacing Clin Electrophysiol 1989; 12 (12):1863-8.

11 Menezes A S Jr \& Dourado J C - Análise de variabilidade dos intervalos RR em pacientes com marcapasso Inos DR e indivíduos normais. Reblampa 1997; 10: 229. 